\title{
Modal Analysis of Partially Coherent Submillimeter-Wave Quasi-Optical Systems
}

\author{
Stafford Withington, Member, IEEE, and J. Anthony Murphy, Member, IEEE
}

\begin{abstract}
We consider the modal analysis of partially coherent submillimeter-wave quasi-optical systems. According to our scheme the cross-spectral density at a plane is expanded as a sum of partially coherent propagating free-space modes. The coherence matrix, the elements of which are determined by evaluating bimodal overlap integrals, completely describes the state of the field at a plane. The coherence matrix can be traced through an optical system to another plane by means of a scattering matrix. Whereas diagonalizing the scattering matrix gives the natural modes of the optical system, diagonalizing the coherence matrix gives the natural modes of the field. We demonstrate the technique by studying the behavior of Gaussian beam telescopes when the field at the source plane is completely incoherent.
\end{abstract}

Index Terms-Submillimeter-wave propagation.

\section{INTRODUCTION}

$\mathbf{W}^{\mathrm{s}}$ E consider how modal techniques that have been developed for the analysis of coherent submillimeterwave optical systems [1]-[3] can be extended to cover the case when the field passing through the system is partially coherent. Partial coherence arises in some form in all problems of practical importance. For example, consider the situation where a submillimeter-wave telescope is used to observe an extended radio astronomical source. Usually, in the case of a heterodyne detector, one would calculate the coupling between the detector and the source by propagating the fully coherent field of the horn "backward" through the optical system onto the sky [4], [5]. There is no reason why, however, the fully incoherent field of the source should not be propagated "forward" through the optical system onto the focal plane. Clearly, in the case of a single-mode detector, the first approach is adequate, but there are many situations where it is not possible to perform detailed calculations unless one has access to the more general description afforded by the second approach-reflect for a while on how one would calculate the performance of a focal-plane array of planar bolometers.

In this paper, we describe a procedure that allows the second-order statistical properties of a field to be traced through a complex system of submillimeter-wave optical components. Unlike Fourier optics [6], the analysis is not limited

Manuscript received November 4, 1997; revised June 8, 1998. This work was supported by Forbairt/Eolas and the Director of the James Clerk Maxwell Telescope, operated by the U.K. Particle Physics and Astronomy Research Council.

S. Withington is with the Cavendish Laboratory, Department of Physics, University of Cambridge, Cambridge, CB3 OHE U.K.

J. A. Murphy is with the Department of Physics, National University of Ireland, Maynooth, Ireland.

Publisher Item Identifier S 0018-926X(98)08885-1. to conjugate Fourier planes and is applicable in cases where significant aberrations are present. The partially coherent field is constructed from a set of coherent diffracting free-space modes. A central feature of the scheme is that the second-order statistical properties of the field are represented completely and, therefore, problems of great complexity can be solved. For example, it is possible to model the behavior of multimode bolometer imaging arrays. Here the state of the whole field is described by a single coherence matrix. Even in the case of a single-mode detector, the unused orthogonal optical modes are excited by noise and this noise appears in the analysis in a natural and elegant manner. Looking to the future, it should be possible to model the correlations that exist between the outputs of a focal plane array when the atmosphere above a radio telescope is varying. At a deeper level, modal analysis provides an understanding of the thermodynamic entropy of a beam in such a way that it should be possible to use maximum entropy techniques to examine the state of a collimated field from intensity measurements alone [7], [8].

\section{Gaussian-Mode Expansion OF THE CROSS-SPECTRAL DENSITY}

We assume that the submillimeter-wave system to be analyzed comprises a sequence of components that interact with and scatter a propagating free-space beam [9], [10]. For partially coherent fields, the quantity we wish to decompose into modes is the cross-spectral density; where, formally, we understand the cross-spectral density to be the time Fourier transform of the mutual coherence function. To this end we assume that the optical system under consideration is one member of an ensemble. If the bandwidth is sufficiently narrow so that the coherence length is very much greater than the physical size of the system, the phase at one point in one member of the ensemble is well defined with respect to the phase at another point in the same member of the ensemble. As in the coherent case, the field can then be written as a modal sum [11]

$$
E^{i}(\mathrm{r}, \omega)=\sum_{m} A_{m}^{i}(\omega) \psi_{m}(\mathrm{r}, \omega)
$$

where $i$ denotes a particular member of the ensemble and frequency dependence has been indicated by $\omega$. We can now represent the cross-spectral density as a bimodal expansion

$$
\begin{aligned}
W\left(\mathbf{r}_{1}^{\prime}, \mathbf{r}_{\mathbf{1}}\right) & =\left\langle E\left(\mathbf{r}_{1}\right) E^{*}\left(\mathbf{r}_{1}^{\prime}\right)\right\rangle \\
& =\sum_{m} \sum_{m^{\prime}} C_{m, m^{\prime}} \psi_{m^{\prime}}^{*}\left(\mathbf{r}_{1}^{\prime}\right) \psi_{m}\left(\mathbf{r}_{\mathbf{1}}\right)
\end{aligned}
$$


where

$$
C_{m, m^{\prime}}=\left\langle A_{m^{\prime}}^{i *} A_{m}^{i}\right\rangle .
$$

We have dropped the explicit reference to frequency, but an integration must be carried if the bandwidth of the system is large.

For convenience we can write the coefficients of the bimodal expansion in matrix form

$$
\mathbf{C}=\left\langle\mathbf{A}^{i} \mathbf{A}^{i * T}\right\rangle
$$

where $\mathbf{A}^{i}$ is the column vector of mode coefficients corresponding to the $i$ th member of the ensemble and $* T$ denotes the conjugate transpose. Once we know the expansion coefficients $\mathbf{C}$ we have characterized the form of the partially coherent field. The propagation of the coherence matrix in the partially coherent case corresponds to the propagation of the mode coefficients in the fully coherent case.

Although physically appealing the above argument is not mathematically rigorous. The problem lies in the fact that in order to generate the modal expansions, we tacitly assumed that we could Fourier transform the time-dependent field of each member of the ensemble and yet it is well known that it is not possible, because of lack of convergence, to Fourier transform the members of a stationary random process. Wolf has considered the modal expansion of three-dimensional (3D) stationary random fields in some detail [12], [13]. The arguments are somewhat lengthy, but the outcome is that modal expansions of the above kind are valid, as physical intuition would suggest. In other words, the expansions themselves are mathematically rigorous, but one has to be extremely careful about the way in which the formalism is proven. The essential point to bear in mind is that one is propagating a statistical property of the field rather than the field itself and this quantity, the cross-spectral density, propagates according to Helmholtz equation. For a greater understanding of these and related issues, the reader is referred to Mandel and Wolf's excellent book [14].

We now need to know how to calculate the bimodal coefficients when the functional form of the cross-spectral density is known. By a simple extension of the usual overlap integral we find that the elements of the coherence matrix are given by

$$
C_{m, m^{\prime}}=\int_{S_{1}} W\left(\mathbf{r}_{\mathbf{1}}^{\prime}, \mathbf{r}_{\mathbf{1}}\right) \psi_{m^{\prime}}\left(\mathbf{r}_{\mathbf{1}}^{\prime}\right) \psi_{m}^{*}\left(\mathbf{r}_{1}\right) d S_{1} d S_{1^{\prime}}
$$

where we have assumed that expansion functions $\psi_{m}$ form an orthonormal set. At the outset we do not know the crossspectral density at every plane, but there is usually some plane over which the cross-spectral density is known.

\section{Natural Modes of a Partially Coherent Field}

We have expanded the cross-spectral density in terms of some arbitrary mode set. We can, of course, transform to some other basis set and describe the field equally well. Of particular interest is the unitary transformation that diagonalizes the coherence matrix. In a sense, the modes found in this way are the natural modes of the field.
The problem of how to expand the cross-spectral density of a 3-D random field in terms of its natural modes has been considered by Wolf [12], [13]. Wolf's approach was to consider Mercer's theorem, which states that if the kernel of a homogeneous Fredholm equation of the second kind is Hermitian and nonnegative definite, then the eigenvalue spectrum is discrete, real, and the eigenvectors form a complete orthonormal set in terms of which the kernel can be expanded. When looking for a bimodal expansion, it is therefore natural to set up an integral equation of the form

$$
\lambda_{i} \phi_{i}\left(\mathbf{r}_{1}\right)=\int W\left(\mathbf{r}_{1}^{\prime}, \mathbf{r}_{1}\right) \phi_{i}\left(\mathbf{r}_{1}^{\prime}\right) d \mathbf{r}_{1}^{\prime}
$$

so that the kernel can be expressed as a weighted sum of eigenfunctions

$$
W\left(\mathbf{r}_{1}^{\prime}, \mathbf{r}_{1}\right)=\sum_{i} \lambda_{i} \phi_{i}^{*}\left(\mathbf{r}_{1}^{\prime}\right) \phi_{i}\left(\mathbf{r}_{1}\right) .
$$

These eigenfunctions $\phi_{i}$ are the natural modes of the field and $\lambda_{i}$ are the associated eigenvalues.

We can now ask what is the relationship between Wolf's natural mode set and the arbitrary set of modes we have used. If we expand the natural modes as a sum of our as yet undefined propagating modes, we have

$$
\phi_{i}\left(\mathbf{r}_{1}\right)=\sum_{n} E_{n}^{i} \psi_{n}\left(\mathbf{r}_{1}\right)
$$

and if we substitute this equation together with the bimodal expansion (2) into the eigenvalue expression (6), we find that

$$
\left[\mathbf{C}-\lambda_{i} \mathbf{I}\right] \mathbf{E}^{i}=0
$$

where now $\mathbf{E}^{i}$ are the mode coefficients of the natural mode $i, \lambda_{i}$ is the associated eigenvalue, and orthonormality of the expansion functions $\psi_{n}$ has been assumed.

To understand the physical meaning of the natural modes, suppose that we have an ensemble of optical systems, where the field associated with each member of the ensemble is constructed from spatially coherent modes, the phases of which are fully incoherent and uniformly distributed with respect to each other. In this case, we have

$$
C_{m m^{\prime}}=\left\langle\left|A_{m^{\prime}}^{i}\right|\left|A_{m}^{i}\right|\right\rangle\left\langle\exp \left[j\left(\theta_{m}^{i}-\theta_{m^{\prime}}^{i}\right)\right]\right\rangle=\lambda_{m} \delta_{m, m^{\prime}} .
$$

The coherence matrix is diagonal as one would expect. Expanding a random process as a sum of orthogonal uncorrelated functions is known as a Karhunen-Loéve expansion and such expansions are important in the related field of adaptive optics [15].

In summary, we can expand the cross-spectral density in terms of any convenient mode set by using the bimodal form of the overlap integral. Because the modes are not chosen in any particular way, correlations will exist between the mode coefficients. If we diagonalize the coherence matrix, we can express the cross-spectral density as a sum of modes which are completely uncorrelated with respect to each other and, therefore, have no definite phase relationship between them. These are the natural modes of the optical field as defined by Wolf. 


\section{COMPLETELy INCOHERENT SOURCES}

Of particular interest is the case where the field at the input plane is fully incoherent and has uniform intensity. This situation occurs, for example, when the beam of a submillimeter-wave telescope is directed toward to a source having uniform brightness. For an incoherent field, we can write

$$
W\left(\mathbf{r}_{1}^{\prime}, \mathbf{r}_{1}\right)=I\left(\mathbf{r}_{1}\right) \delta\left(\mathbf{r}_{1}-\mathbf{r}_{1}^{\prime}\right) .
$$

Substituting this expression into the bimodal overlap integral, we find

$$
C_{m, m^{\prime}}=\int_{S_{1}} I\left(\mathbf{r}_{1}\right) \psi_{m^{\prime}}\left(\mathbf{r}_{1}\right) \psi_{m}^{*}\left(\mathbf{r}_{1}\right) d S_{1} .
$$

Now if the source has uniform brightness

$$
C_{m, m^{\prime}}=I_{o} \delta_{m, m^{\prime}} \cdot
$$

That is to say, all of the modes are excited equally and independently. It can be shown that because of completeness, this must be true regardless of what mode set is used. Physically, one is saying that the resultant field cannot contain any spatial information. In matrix form, we have for a uniform incoherent source

$$
\mathbf{C}=I_{o} \mathbf{I}
$$

where $\mathbf{I}$ is the identity matrix. Clearly, if the brightness over the plane of the source is not uniform, correlations must exist between modes even though the source itself is incoherent. This behavior is to be expected classically because the van Cittert-Zernike theorem tells us that correlations exist in the far field of a source of finite size, even when the source itself is incoherent.

\section{Propagating the Coherence Matrix}

It is well known that a coherent field can be traced through a submillimeter-wave optical system by multiplying the mode coefficients of the incident beam by a scattering matrix. Moreover, if the optical system comprises a number of optical components then the scattering matrix associated with the overall system is simply the product of the scattering matrices associated with the individual components [2]. We now need to ask how a partially coherent field can be traced through a submillimeter-wave optical system.

We know that for each member of the ensemble we can propagate the field according to the usual Gaussian modescattering matrix $\mathbf{S}$. Hence, if the field at the input plane has mode coefficients $\mathbf{A}^{i}$ then the field at the output plane has mode coefficients $\mathbf{B}^{i}$ where

$$
\mathbf{B}^{i}=\mathbf{S A}^{i} .
$$

The coherence matrix at the output plane therefore becomes

$$
\left\langle\mathbf{B}^{i} \mathbf{B}^{i * T}\right\rangle=\mathbf{S}\left\langle\mathbf{A}^{i} \mathbf{A}^{i * T}\right\rangle \mathbf{S}^{* T}
$$

or

$$
\mathbf{D}=\mathbf{S C S}^{* T}
$$

where $\mathbf{D}$ is the coherence matrix at the output plane, $\mathbf{S}$ is the usual coherent mode-scattering matrix, and $\mathbf{C}$ is the coherence matrix at the input plane. Numerically, the procedure is straightforward and the scattering matrix only has to be calculated once for a given optical system. The scattering matrix contains all of the information necessary to propagate a coherent or incoherent field. Notice also that if the optical system under consideration is varying with time, as would be the case for a turbulent atmosphere above a radio telescope, then the ensemble average would include the time-varying scattering matrix also.

\section{General Considerations}

It is vitally important to appreciate that the coherence matrix characterizes the modal properties of the field, whereas the scattering matrix characterizes the modal properties of the optical system: the two are quite distinct. We can diagonalize the coherence matrix to get the mode coefficients of the natural modes of the field. The eigenvalues then give the amount of power in each mode. When performing numerical calculations on a practical system, we do not know and are not, therefore, able to use the natural modes of the field (in general, the natural modes have complex functional forms) but nevertheless we should, from the point of view of numerical efficiency, use a convenient set of modes that diagonalizes the coherence matrix as nearly as possible.

In a previous paper [2], we discussed the diagonalization of the scattering matrix. In this case, the eigenvectors give the mode coefficients of coherent field distributions that pass through the optical system unchanged, and the eigenvalues give the loss associated with the propagation of these fields. These modes are the normal modes of the optical system and, in general, any incoming field distribution can be expanded in terms of these modes and propagated simply by multiplying by the eigenvalues. For convenience, we should, for coherent fields, choose a mode set that propagates easily and yet which diagonalizes as nearly as possible the scattering matrix. For partially coherent fields, the natural modes of the optical system are not the same as the natural modes of the field and we can ask whether it is more reasonable to choose a mode set that near diagonalizes the coherence matrix or a mode set that near diagonalizes the scattering matrix.

To answer this question, it is particularly revealing to ask what happens when an optical system, described by a scattering matrix $\mathbf{S}$, is illuminated by a uniform fully incoherent source. We know that for any set of modes the coherence matrix of the source is diagonal and, therefore, the field at the output plane is given by

$$
\mathbf{D}=I_{o} \mathbf{S I S}^{* T} .
$$

It is clear that although the field at the input plane is fully incoherent, the field at the output plane has coherence induced on it due to mode filtering. The introduction of coherence is evidenced by the appearance of off-diagonal terms in the output-coherence matrix. We know, however, that there is some mode set that diagonalizes the scattering matrix and, in this case, the coherence matrix at the output plane is also 
diagonal. Hence, if an optical system is illuminated by a uniform incoherent field, the natural modes of the resultant field are the same as the natural modes of the optical system itself. We will, therefore, make the physically reasonable assumption that we should choose a mode set that diagonalizes as nearly as possible the scattering matrix of the system, regardless of the state of coherence of the incoming field.

It is also interesting to notice that spatial coherence builds up as modes are filtered. We know that when a low throughput submillimeter-wave optical system is illuminated by a coherent source, the output of the system will tend to the lowest-order eigenmode regardless of the precise nature of the incoming field and this is why low throughput submillimeterwave optical systems always, somewhat conveniently, tend to produce Gaussian beams. In the case of incoherent illumination the situation is similar: as more and more low throughput components are added, the output becomes a fully coherent Gaussian beam.

A further important and useful consideration is that the number of significant nonzero eigenvalues found when diagonalizing the scattering matrix gives the number of degrees of freedom of the optical system. The number of nonzero eigenvalues found when diagonalizing the coherence matrix gives the number of degrees of freedom of the field. Clearly, the number of degrees of freedom of the output field can only be as many as the optical system and the two will be the same when the incident field is fully incoherent. In the case where the incoming field is fully coherent, the coherence matrix will have only one nonzero eigenvalue, a feature that can be traced to the fact that the elements of the coherence matrix factorize. In general, this will mean that because the coherence matrix has $N^{2}$ elements it will not be possible to diagonalize. The solution to this apparent paradox-that is to say we have a matrix with $N^{2}$ elements which seems to have only one nonzero eigenvalue-is that the modes of the optical system will all, at some level, be excited by noise. In fact, it can easily be seen that we can add a noise coherence matrix to a fully coherent coherence matrix to get a matrix that can be diagonalized and if the signal-to-noise ratio is high, one eigenvalue will be much greater than all of the others. The ability to study the signal and noise properties of a field through diagonalization is a particularly useful technique. For example, if the field "produced" by a complete array of detectors [16] is described by a single coherence matrix, the eigenvalues will give the relative sensitivities of the pixels.

\section{SCATTERING MATRIX OF THE GAUSSIAN BEAM TELESCOPE}

Although the technique described can be used to analyze the behavior of almost any system, in this section, we shall apply the theory to the Gaussian beam telescope [1]. Not only does the Gaussian beam telescope exhibit features that are present in all systems, but it also produces results that can be interpreted easily in terms of classical analysis [17], [18]. In order not to cloud the central features of the model, we shall work in one dimension, but the extension to two dimensions is straightforward.

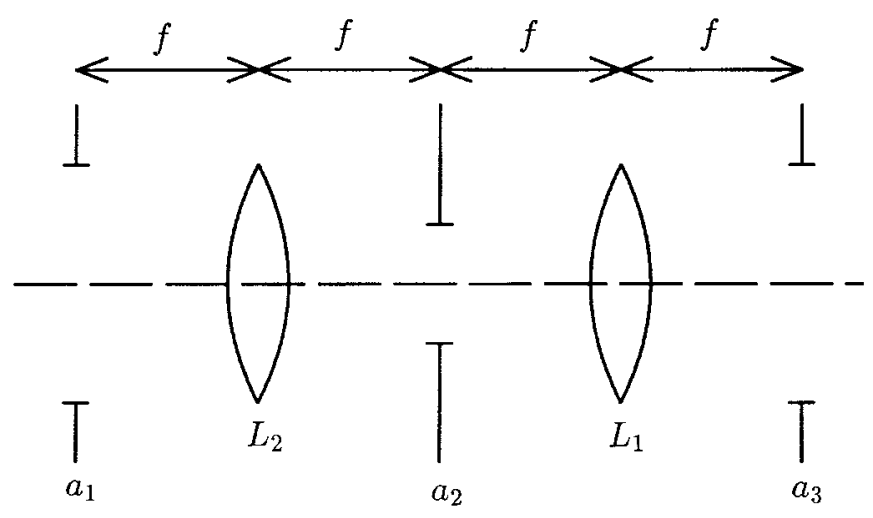

Fig. 1. A Gaussian beam telescope.

A diagram of a Gaussian beam telescope is shown in Fig. 1. This arrangement is important because the field at the input plane is imaged onto the field at the output plane in a frequency-independent way. We have included two apertures in order to limit the throughput of the system, as would be the case for any real system with finite size components.

To begin, we must calculate the scattering matrix. Let us represent the fields in the regions between the components in terms of propagating Gaussian-Hermite modes. Each mode has the form

$$
\psi_{m}(x)=\left(\frac{\sqrt{2}}{w}\right)^{1 / 2} h_{m}\left(\frac{\sqrt{2} x}{w}\right) \exp \left[ \pm j \theta \mp \frac{j \pi x^{2}}{\lambda R} \mp j k z\right]
$$

where

$$
h_{m}(u)=\frac{H_{m}(u) \exp \left[-\frac{u^{2}}{2}\right]}{\left(\sqrt{\pi} 2^{m} m !\right)^{1 / 2}}
$$

and

$$
\theta=(m+1 / 2) \frac{z}{z_{c}}
$$

also $H_{m}(u)$ is the Hermite polynomial of order $m$ in $u$. The functions $h_{m}(u)$ are orthonormal in the sense that

$$
\int_{-\infty}^{+\infty} h_{m}(u) h_{n}(u) d u=\delta_{m n}
$$

In these equations, the symbols have their usual meanings [1]: $w$ characterizes the scale size of the beam at a plane; $R$ characterizes the large-scale radius of curvature of the phase front; and $\theta$, the phase slippage between modes, characterizes the form of the field as the beam propagates and diffracts. $z_{c}$ is the Rayleigh distance. As has already been described in some detail [2], a mode set is not completely defined until the size $w_{o}$ and position of the waist are given. We do know that for a Gaussian beam telescope, the large-scale phase front of the field at the focal planes is flat and, therefore, we can place the waists at these positions.

Having decided on the mode set, we know that the scattering matrix of the whole system is just the product of the scattering matrices of the individual components. The components in this case are the apertures and the free-space paths; as usual, the 
focusing effects of the ideal lenses are taken up by choosing the mode sets appropriately; that is to say the waists at all but the input plane are chosen according to the usual singlemode Gaussian beam analysis. If we adopt this scheme, there is no modal scattering associated with the ideal lenses. If offaxis mirrors are incorporated then the appropriate scattering matrices must, of course, be used [19].

The size of the waist at the input plane is still undetermined and although any waist would produce a complete mode set, some particular input waists will be numerically more efficient than others. In a previous paper [2], we discussed (at some length) that the waist should be chosen to diagonalize as nearly as possible the scattering matrix. In fact, in that paper, we diagonalized the scattering matrix to recover the eigenmodes and eigenvalues, which are known from classical analysis to be prolate spheroidal wavefunctions. We are, of course, merely talking about efficiency of convergence and so the precise choice is not critical anyway.

Hence, as before [2], we take the input waist of the optimum mode set to be

$$
w_{1}=\left[\frac{\lambda f a_{1}}{\pi a_{2}}\right]^{1 / 2}
$$

where $a_{1}$ and $a_{2}$ are the half widths of the apertures, and $f$ is the focal length of the lenses. The number of modes that should be used in the expansion is approximately the Fresnel number $c$ where

$$
c=\frac{2 \pi a_{1} a_{2}}{f \lambda}
$$

We are now in a position to derive the scattering matrix. First, we must calculate the scattering matrices of the apertures. By evaluating the field overlap integral over the output plane of each aperture [20], [21], we find that the scattering matrices are given by

$$
S_{m, n}=\int_{-\sqrt{2} k_{t}}^{+\sqrt{2} k_{t}} h_{n}(u) h_{m}(u) d u
$$

where $k_{t}=a / w$ is the normalized truncation. Because the mode set is, by definition, chosen so that the truncation at each stop is the same, we can easily write

$$
S_{m, n}=\int_{-\sqrt{c}}^{+\sqrt{c}} h_{n}(u) h_{m}(u) d u .
$$

Hence, once we have chosen the Fresnel number, the scattering matrices of the two apertures are the same and given by the above expression. We could evaluate this matrix numerically, but we have found the following recurrence relationships useful. First, we calculate the lowest-order element through

$$
S_{00}=\operatorname{erf}(\sqrt{c})
$$

where $\operatorname{erf}(u)$ is the error function. Then, for all $n+1$ odd we have

$$
S_{0,(n+1)}=S_{(n+1), 0}=0
$$

and for all $n+1$ even we have

$$
S_{0,(n+1)}=S_{(n+1), 0}=-\left(\frac{2}{n+1}\right)^{1 / 2} h_{0}(\sqrt{c}) h_{n}(\sqrt{c}) .
$$

Also, for $(m+1)+(n+1)$ odd

$$
S_{(m+1),(n+1)}=S_{(n+1),(m+1)}=0
$$

and for $(m+1)+(n+1)$ even

$$
\left(\frac{m+1}{n+1}\right)^{1 / 2} S_{m, n}-\left(\frac{2}{n+1}\right)^{1 / 2} h_{m+1}(\sqrt{c}) h_{n}(\sqrt{c}) .
$$

These equations show that power does not scatter between odd- and even-ordered modes as would be expected.

We also need the scattering matrices of the free-space paths. These are easily found because we know that there is a Fourier transform relationship between the fields at the focal planes or, equivalently, the phase slippage is $\pi / 2$ [2]. The scattering parameters of the free-space paths become

$$
S_{m, n}=\left[\cos \left(\frac{\pi m}{2}\right)+j \sin \left(\frac{\pi m}{2}\right)\right] \delta_{m, n} .
$$

If we denote the scattering matrices of the free-space paths by $\mathbf{S}_{f}$ and the scattering matrices of the apertures by $\mathbf{S}_{a}$ then the scattering matrix of the whole Gaussian beam telescope is simply given by the product

$$
\mathbf{S}=\mathbf{S}_{f} \mathbf{S}_{a} \mathbf{S}_{f} \mathbf{S}_{a}
$$

This matrix is extremely simple to generate using the above equations and it is remarkable that it completely characterizes the coherent and partially coherent behavior of the system.

\section{PARTIAL COHERENCE AND THE GAUSSIAN BEAM TELESCOPE}

Now that we have generated the scattering matrix, we can investigate its response to various different kinds of excitation. Before studying the propagation of partially coherent radiation, it is worthwhile verifying the integrity of the scattering matrix by investigating the coherent behavior.

First, we can plot the point-spread function. Calculating the mode coefficients of the one-dimensional (1-D) delta function by evaluating the overlap integral and then substituting the mode coefficients into the modal expansion of the field, we find that the point-spread function is given by

$$
E_{\mathrm{psf}}\left(x, x^{\prime}\right)=\left(\frac{\sqrt{2}}{w}\right) \sum_{m} \sum_{n} S_{m, n} h_{n}\left(\frac{\sqrt{2} x^{\prime}}{w}\right) h_{m}\left(\frac{\sqrt{2} x}{w}\right)
$$

where $S_{m, n}$ are the elements of the scattering matrix and $x^{\prime}$ is the position of the delta function in the input plane. Clearly, this expression applies to any system for which the scattering matrix is known. In Fig. 2, we show the point-spread functions of Gaussian beam telescopes having Fresnel numbers of 4, 8, and 16.

Rather than plotting the point-spread function for different input positions, it would be convenient to have some simple measure of its form. Classically, the crudest method use to use the Strehl ratio, where the Strehl ratio is defined as the height of the central peak normalized to the height of this peak when excited by a signal at the central position. In the 


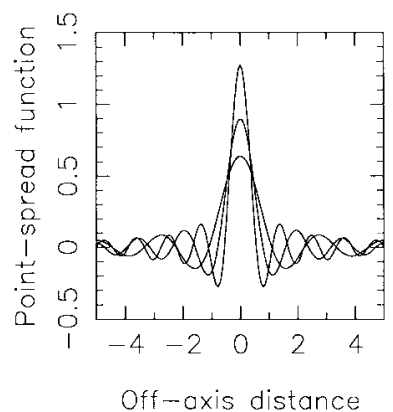

Fig. 2. The point-spread functions of Gaussian beam telescopes having Fresnel numbers of 4,8 , and 16 . The largest peak corresponds to the largest Fresnel number. The off-axis distance is normalized to the waist: $x / w .48$ modes were used to construct these plots.

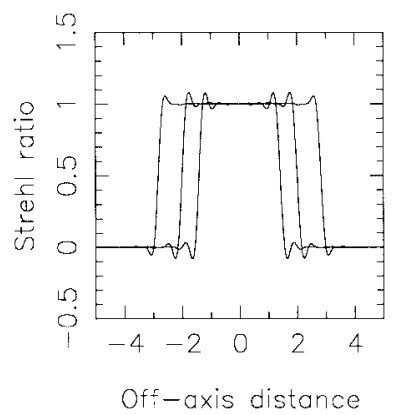

Fig. 3. The Strehl ratios of Gaussian beam telescopes having Fresnel numbers of 4,8 and 16. The largest aperture corresponds to the largest Fresnel number. In each case, the Strehl ratio is shown as a function of normalized $x / w$ off-axis distance and 48 modes were used to construct these plots.

case of Gaussian modes it is particularly easy to calculate the Strehl ratio; it is given by

$$
S=\frac{\sum_{m} \sum_{n} S_{m, n} h_{n}\left(\frac{\sqrt{2} x}{w}\right) h_{m}\left(\frac{-\sqrt{2} x}{w}\right)}{\sum_{m} \sum_{n} S_{m, n} h_{n}(0) h_{m}(0)}
$$

Notice that the sign on the argument on one of the Hermite functions has been changed to take into account the fact that the image is inverted. In Fig. 3, we show the Strehl ratio as a function of normalized off-axis position for Fresnel numbers of 4,8 , and 16 .

The Strehl ratio is independent of position over the whole of the field of view and this independence occurs simply because we did not include any abberations in our system. Some ringing can be seen at the edge of the input aperture. This ringing is not an optical effect, but exists merely because we are trying to represent the abrupt disappearance of the input field behind the input aperture with a finite number of modes. This behavior is the modal equivalent of Gibb's phenomenon. The most impressive feature of these plots is that they demonstrate that a small number of modes can represent the behavior of the system over the whole of the field of view and this has significant implications for modeling the behavior of submillimeter-wave imaging arrays.

We would now like to investigate the behavior of the system when a fully incoherent source with a Gaussian intensity distribution is applied. To perform this calculation, we require the coherence matrix. Using the 1-D form of the bimodal

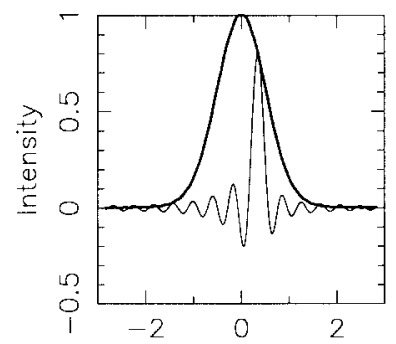

(a)

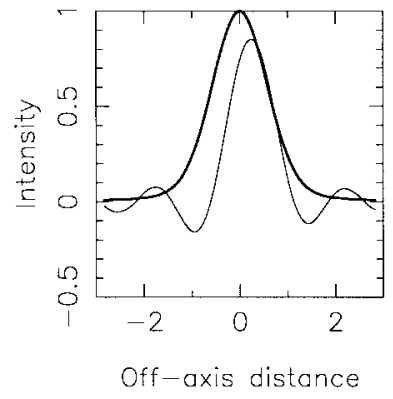

(c)

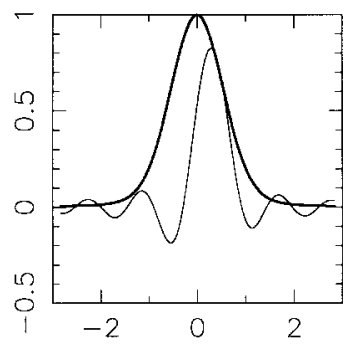

(b)

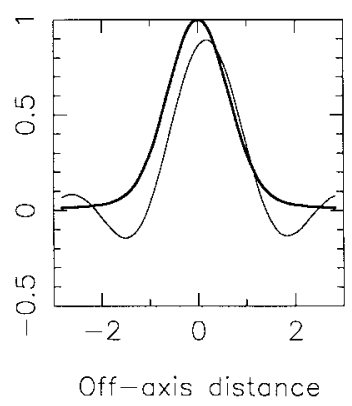

(d)
Fig. 4. (a) The Gaussian intensity (solid line) and cross-spectral density (faint line) of the field at the input plane of a Gaussian beam telescope. Sixty modes were used to synthesize a nearly fully incoherent field. The Gaussian input field has an effective normalized width of 0.707. In (b), (c), and (d), we show the intensity and cross-spectral density at the output plane when the Fresnel number is 16,8 , and 4 , respectively.

overlap integral and a cross-spectral density of the form

$$
\begin{aligned}
W\left(x^{\prime}, x\right) & =I(x) \delta\left(x-x^{\prime}\right) \\
& =K^{2} \exp \left[-\frac{2\left(x-x_{o}\right)^{2}}{\sigma^{2}}\right] \delta\left(x-x^{\prime}\right)
\end{aligned}
$$

we find

$$
\begin{aligned}
C_{m, n}= & K^{2}\left(\frac{\sqrt{2}}{w}\right) \int_{-\infty}^{+\infty} \exp \left[-\frac{2\left(x-x_{o}\right)^{2}}{\sigma^{2}}\right] \\
& \times h_{n}\left(\frac{\sqrt{2} x}{w}\right) h_{m}\left(\frac{\sqrt{2} x}{w}\right) d x .
\end{aligned}
$$

In Fig. 4, we show the intensity of the field at the input and output planes when the Fresnel number is 4, 8, and 16 . The normalized width of the effective input field distribution, $\sigma / w_{1}$, was taken to be 0.707 . Superimposed on each plot is the cross-spectral density when the normalized reference position is $x / w_{3}=0.35$; we could, trivially, have chosen any other reference position and the result would have been essentially the same.

For the purpose of presenting (in the diagram) a highly incoherent input field, we used 60 modes, but this large number of modes is not actually needed for the analysis. The main feature is, as would be expected, a slight spreading of the output intensity with decreasing Fresnel number and an increasing degree of coherence. What is not seen, because of normalization, is the large amount of power lost, which would not be the case for coherent illumination.

In addition to these plots, it is also convenient to look at the behavior when the input is a flat incoherent field of finite 


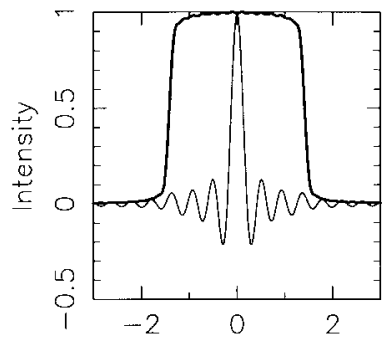

(a)

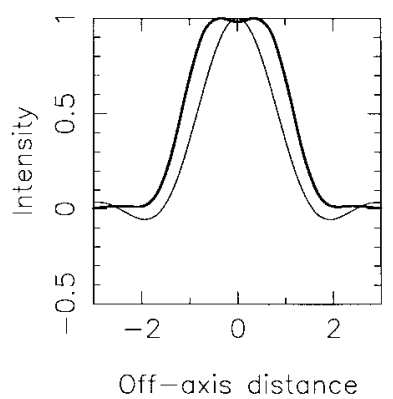

(c)

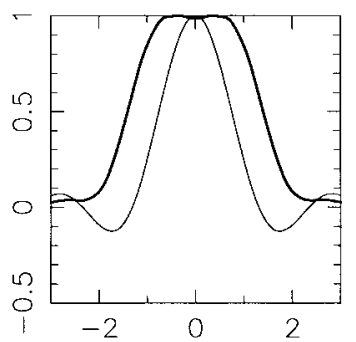

(b)

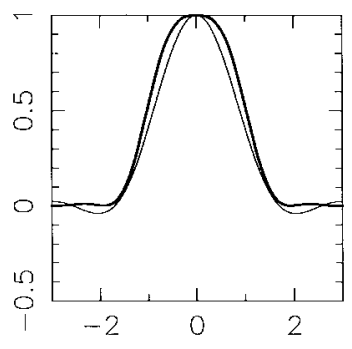

Off-axis distance

(d)
Fig. 5. (a) The top-hat intensity (solid line) and cross-spectral density (faint line) of the field at the input plane of a Gaussian beam telescope. Sixty modes were used to synthesize a nearly fully incoherent field. The top-hat input field has an effective normalized half-width of $\sqrt{2}$. In (b), (c), and (d), we show the intensity and cross-spectral density at the output plane when 1 , 2, and 4 Gaussian beam telescopes, each having a Fresnel number of four, are connected in series.

extent. In this case, the elements of the coherence matrix are given by

$$
C_{m, n}=\left(\frac{\sqrt{2}}{w}\right) \int_{-b}^{+b} h_{n}\left(\frac{\sqrt{2} x}{w}\right) h_{m}\left(\frac{\sqrt{2} x}{w}\right) d x
$$

where $b$ is the extent of the field. In the case where $b \rightarrow \infty$, the coherence matrix becomes diagonal, as expected. In Fig. 5 we show the intensity and cross-spectral density at the input and output planes when one, two, and four Gaussian beam telescopes having Fresnel numbers of four are connected in series. The scattering matrix of the overall system is simply the scattering matrix of one telescope raised to the appropriate power. It can be seen how coherence builds up due to mode filtering and, after four passes, a nearly fully coherent Gaussian field is produced despite having started with fully incoherent top hat. Indeed, this is precisely the way in which coherence builds up in a laser cavity [22]-[24]. Here we have simply used this well-known phenomenon to demonstrate the scatteringmatrix technique.

As a final more complicated demonstration, consider placing a two-element array of detectors in the focal plane. We recognize that the focal plane is not completely populated and represent the missing elements by microwave absorber. Moreover, in order to retain the simple 1-D geometry, we assume that each element in the array is an open-ended rectangular waveguide. Each waveguide is single moded, but as a pair they essentially form a two-moded detector: we could, of course, have used a single-overmoded piece of waveguide to produce a few-moded detector. We now wish to calculate the statistical properties of the field at the output plane.
To solve the problem, we require the coherence matrix of the whole field at the input plane. It is trivial to show that if we combine two fields that are generated incoherently, the overall coherence matrix is the sum of the two individual coherence matrices regardless of the states of coherence of the individual fields. This theory has application when a submillimeter-wave beam is truncated by a lossy aperture that injects noise of its own. Because in our case, the three principle fields (two fully coherent offset cosine fields and one fully incoherent sheet with a hole) are mutually uncorrelated, we simply need to find the individual coherence matrices and then add them together. Notice that we have chosen the example specifically so that we have to combine fully coherent and fully incoherent input fields.

The first task is to find the coherence matrix of a uniform sheet of microwave absorber with a hole in the middle. There are various ways of approaching this problem, but first consider placing another sheet of absorber behind the hole so as to block it off. If the two absorbers are at the same temperature, then we see a black surface having a coherence matrix of $I_{o} \mathbf{I}$, where $I_{o}$ is a multiplying factor proportional to the radiometric temperature; actually, we know that the power radiated is

$$
P=\operatorname{Tr}(\mathbf{C})=\sum_{i} C_{i i}=N k T B=N I_{o}
$$

where $N$ is the total number of modes and $B$ is the bandwidth. We also know that the contributions from the individual screens are uncorrelated and so we can write

$$
\mathbf{D}=I_{o} \mathbf{I}-\mathbf{C}
$$

where $\mathbf{C}$ and $\mathbf{D}$ are the coherence matrices of the front and back screens, respectively. Now the contribution from the back screen is given by (18) and, therefore

$$
\mathbf{D}=I_{o}\left(\mathbf{I}-\mathbf{S S}^{* T}\right) .
$$

Hence, we can easily calculate the coherence matrix of an absorbing aperture of any shape if the scattering parameters are known. This theorem is vitally important and it is closely related to Bosma's theorem [25] for microwave networks. Actually, we believe that it must be possible to calculate the coherence matrix of any passive optical component once the modal scattering parameters are known. Also notice that the noise performance of the optical component is completely described by a set of complex temperatures in much the same way that the noise performance of a microwave network is completely described by a set of complex temperatures. Moreover, these could be transformed to an equivalent set of noise parameters at the input of the complete optical system if required [26].

Rather than using the scattering matrix to derive $\mathbf{D}$, as described above, we can find $\mathbf{C}$ directly. By using (12) and after some manipulation, we have

$$
C_{m n}=\int_{-\sqrt{2} k_{t}^{\prime}}^{+\sqrt{2} k_{t}^{\prime}} h_{m}(u) h_{n}(u) d u
$$




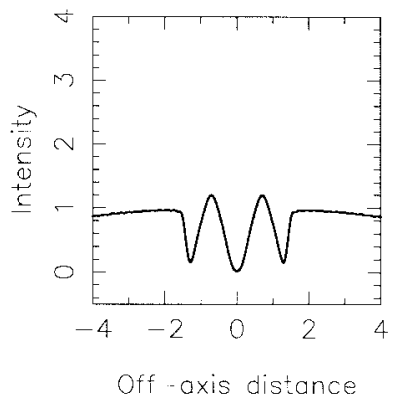

(a)

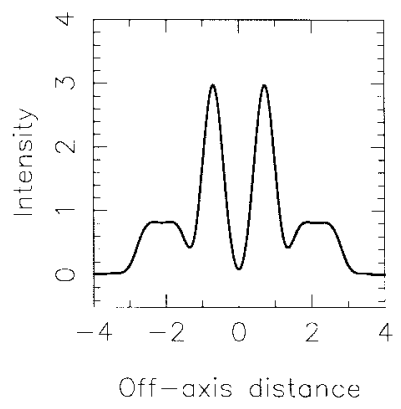

(b)
Fig. 6. The intensity distributions at the (a) input and (b) output planes of a Gaussian beam telescope having a Fresnel number of 16 when the input field is produced by an array of two single-moded open-ended rectangular waveguides surrounded by a sheet of microwave absorber. The inner edge of the absorber is at a normalized off-axis distance of $\sqrt{2}$.

but we have already derived a set of recursion relationships for constructing this matrix [see (27)-(31)]. Recurrence relationships are also available in the case of circular apertures when Gaussian-Laguerre modes are used [20], [21].

We also need to calculate the coherence matrix associated with each offset waveguide. For a coherent field, we simply have

$$
\mathbf{C}=\mathbf{A A}^{* T}
$$

and, therefore, the coherence matrix of any coherent field can be calculated once the ordinary mode coefficients are known. The mode coefficients of many of the common horns have been derived in the literature [27], [28]. For the cosine field associated with open-ended waveguide it can be shown that

$$
A_{m}=\left(\frac{\sqrt{2}}{k_{t}^{\prime}}\right)^{1 / 2} \int_{-\sqrt{2} k_{t}^{\prime} 0}^{0 \sqrt{2} k_{t}^{\prime}} \cos \pi\left(\frac{u}{\sqrt{2} k_{t}^{\prime}} \pm \frac{1}{2}\right) h_{m}(u) d u
$$

where we have normalized the power in each waveguide mode to unity. The first set of limits, which corresponds to one horn, is associated with the positive sign and the second set, which corresponds to the other horn, with the negative sign.

In Fig. 6, we show the input and output intensity distributions when the composite field, as described above, is fed into a Gaussian beam telescope having a Fresnel number of 16. A total of 60 modes were used to produce a highly incoherent input field and the edge of the absorbing aperture was placed at a normalized off-axis distance of $a_{1}^{\prime} / w=\sqrt{2}$. It can be seen that on the input side, the two coherent waveguide fields are surrounded by the highly incoherent radiation from the absorber. On the output side the two waveguide fields reappear (actually they are swapped over although it is not seen in this plot) and the field from the absorber is truncated on the outer edge due to the finite field-of-view of the optical system. Another feature is that the incoherent background has been reduced in intensity with respect to the coherent field and this is simply the well-known aperture-stop effect. If one plots the mutual coherence function it also becomes clear that the output field is partially coherent and also the number of coherence areas in the output plane is half of the Fresnel number. Although we have only used a simple scheme for demonstrating the method, it is remarkable that all of this information is contained in just one composite matrix.

\section{CONCLUSION}

We have described a procedure for calculating the behavior of partially coherent submillimeter-wave quasi-optical systems. The technique, like its coherent equivalent, is based on the ability to scatter power between free-space modes at optical components. Rather than describing the propagating coherent field by a vector, the second-order statistical properties of the partially coherent field are described by a matrix. Because the description of the field is more complete problems of great complexity can be solved. In the paper, we demonstrated the procedure by propagating the field from a pair of horns, surounded by microwave absorber, through a Gaussian beam telescope. In practice there seems to be no reason why the technique cannot be applied to practical systems incorporating many awkwardly placed optical elements. The modeling of submillimeter-wave multimode bolometer imaging arrays is a particularly topical issue at the present time. The next stage in our work is to assess the application of this technique to these practical problems. The work will need extending from one to two dimensions, but we do not see any major problem here, particularly when one considers the alternative to be the full diffraction integral analysis of the system for every incoherent exciting mode. In some ways, one of the great strengths of the formalism is the conceptual insight it gives into the way partially coherent submillimeter optical systems behave.

\section{REFERENCES}

[1] P. F. Goldsmith, "Quasioptical techniques at millimeter and submillimeter wavelengths," in Infrared and Millimeter Waves, K. J. Button, Ed. New York: Academic, 1982, vol. 6, ch. 5.

[2] S. Withington and J. A. Murphy, "Multimode Gaussian optics," in Proc. 3rd Int. Workshop Terahertz Electron., Zermatt, Switzerland, Aug. 1995, pp. $1676-1690$.

[3] D. H. Martin and J. W. Bowen, "Long-wave optics," IEEE Trans. Microwave Theory Tech., vol. 41, pp. 1678-1690, Oct. 1993.

[4] R. Padman, J. A. Murphy, and R. E Hills, "Gaussian mode analysis of Cassegrain antenna efficiency," IEEE Trans. Antennas Propagat., vol. AP-35, pp. 1093-1103, Oct. 1987.

[5] J. A. Murphy, "Aperture efficiencies of large axisymmetric reflector antennas fed by conical horns," IEEE Trans. Antennas Propagat., vol. AP-36, pp. 570-575, Apr. 1988.

[6] J. W. Goodman, Introduction to Fourier Optics. New York: McGrawHill, 1968.

[7] S. Withington and K. G. Isaak, "Phase retrieval at submillimeter wavelengths using Gaussian Hermite modes," in Proc. 4th Int. Symp. Space Terahertz Technol., Los Angeles, CA, Apr. 1993, pp. 199-210.

[8] A. Cutolo, A. Esposito, T. Isernia, R. Pierri, and L. Zeni, "Characterization of the transverse modes in a laser beam: Analysis and application to a Q-switched Nd:YAG laser," Appl. Opt., vol. 31, pp. 2723-2733, 1992.

[9] R. Blundell and C.-Y. E. Tong, "Submillimeter receivers for radio astronomy," in Proc. IEEE, vol. 80, pp. 1702-1720, Nov. 1992.

[10] P. F. Goldsmith, "Quasioptical techniques," in Proc. IEEE, vol. 80, pp. 1729-1747, Nov. 1992.

[11] J. A. Murphy and A. Egan, "Examples of Fresnel diffraction using Gaussian modes," Eur. J. Phys., vol. 14, pp. 121-127, 1993.

[12] E. Wolf, "New theory of partial coherence in the space-frequency domain. Part I: Spectra and cross spectra of steady-state sources," $J$. Opt. Soc. Amer., vol. 72, pp. 343-351, 1982.

[13] E. Wolf, "New theory of partial coherence in the space-frequency domain-Part II: Steady-state fields and high-order correlations," J. Opt. Soc. Amer. A., vol. 3, pp. 76-85, 1986. 
[14] L. Mandel and E. Wolf, Optical Coherence and Quantum Optics. Cambridge, U.K.: Cambridge Univ. Press, 1995.

[15] F. Roddier, "The problematic of adaptive optics design," in Adaptive Optics for Astronomy, D. M. Alloin and J.-M. Mariotti, Eds. Amsterdam, The Netherlands: Kluwer, 1994, pp. 89-112.

[16] D. T. Emerson and J. M. Payne, "Multifeed systems for radio telescopes," Astronom. Soc. Pacific Conf. Ser., Tucson, AZ, May 1994, vol. 75.

[17] G. T. D. Francia, "Degrees of freedom of an image," J. Opt. Soc. Amer., vol. 59, pp. 799-804, 1969

[18] M. Bendinelli, A. Consortini, L. Ronchi, and B. R. Frieden, "Degrees of freedom, and eigenfunctions for the noisy image," J. Opt. Soc. Amer., vol. 64, pp. 1498-1502, 1974.

[19] S. Withington, J. A. Murphy, and K. G. Isaak, "Representation of mirrors in beam waveguides as inclined phase-transforming surfaces," Infrared Phys. Technol., vol. 36, no. 3, pp. 723-734, 1995.

[20] J. A. Murphy, A. Egan, and S. Withington, "Truncation in beam waveguides," IEEE Trans. Antennas Propagat., vol. 41, pp. 1408-1413, Oct. 1993.

[21] J. A. Murphy, S. Withington, and A. Egan, "Mode conversion at diffracting apertures in millimeter and submillimeter-wave optical systems," IEEE Trans. Microwave Theory Tech., vol. 41, pp. 1700-1702, Oct. 1993.

[22] E. Wolf, "Spatial coherence in resonant modes in a maser interferometer," Phys. Lett., vol. 3, pp. 166-168, 1963.

[23] D. C. W. Morley, D. G. Schofield, L. Allen, and D. G. C. Jones, "Spatial coherence and mode structure in the He-Ne laser," Brit. J. Appl. Phys., vol. 18, pp. 1419-1422, 1967.

[24] W. Streifer, "Spatial coherence in periodic systems," J. Opt. Soc. Amer., vol. 56, pp. 1481-1489, 1966.

[25] H. Bosma, "On the theory of linear noisy systems," Philips Res. Rep. Suppl., no. 10, 1967.

[26] S. Withington, "Scattered noise waves in millimeter wave networks," Microwave J., vol. 32, pp. 169-178, 1989.

[27] S. Withington and J. A. Murphy, "Analysis of diagonal horns through
Gaussian-Hermite modes," IEEE Trans. Antennas Propagat., vol. 40, pp. 198-206, Feb. 1992

[28] R. J. Wylde, "Millimeter-wave Gaussian beam-mode optics and corrugated feed horns," Proc. Inst. Elect. Eng., vol. 131, pt. H, pp. 258-262, 1984.

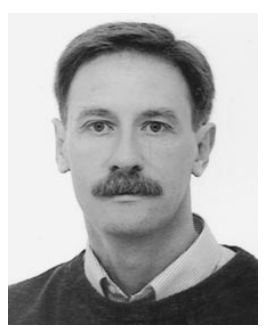

Stafford Withington (M'89) is an Assistant Director of Research in the Radio Astronomy Group of the Cavendish Laboratory, University of Cambridge, U.K. He is also a Fellow of Downing College, Cambridge, where he is Graduate Tutor and Director of Studies in Engineering. His main area of interest is in the submillimeter-wave band, where he works on optics, detectors, and low-noise instrumentation for astronomy.

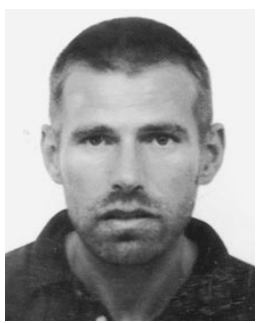

J. Anthony Murphy (M'88) received the B.Sc. and M.Sc. degrees in experimental physics from University College, Cork, Ireland, the M.S. degree in physics from the California Institute of Technology, and the Ph.D. degree in physics from Cambridge University, U.K., in 1977, 1979, 1981, and 1986, respectively.

$\mathrm{He}$ is currently a Senior Lecturer in Experimental Physics at St. Patrick's College (National University of Ireland), Maynooth, Ireland. His main research interests are in submillimeter-wave optics and submillimeter-wave receiver development. 